\title{
Betting big on an AIDS vaccine
}

$\mathbf{T}$ he US National Institutes of Health (NIH) is launching a major new initiative intended to speed progress toward an effective AIDS vaccine, which could funnel more than $\$ 300$ million into the field over the next seven years. The money will fund the establishment of a 'virtual' Center for HIV/AIDS Vaccine Immunology (CHAVI), to be directed by a small team of scientists who will develop a focused program to tackle specific immunological problems in HIV vaccine research. But the initiative has stirred up skepticism among some HIV researchers (see News Feature, page 587). Is such a huge influx of money to a single team the best approach to spark the scientific breakthrough that is required to develop an effective vaccine? The strategy is a gamble and the NIH should hedge its bets and ensure that its new initiative will not, in future years, diminish the existing opportunities for investigator-initiated research.

There is no obvious solution to the problem of how best to tackle the scientific hurdles facing an effective AIDS vaccine. But with no sign of a breakthrough despite two decades of HIV vaccine research, it is certainly warranted to ask whether alternative ways of conducting research might garner greater success. And in this respect, the HIV vaccine community has been galvanized over the last few years. In February 2005, the Global HIV Vaccine Enterprise-an alliance of international vaccine experts, spearheaded by the Bill and Melinda Gates Foundation — published a strategic blueprint, clearly articulating the key obstacles that are impeding progress toward an effective vaccine and outlining some practical goals that need to be achieved (PLoS Med. 2, e25; 2005).

At the crux of the Global Enterprise's blueprint is a call for greater collaboration between scientists. Specifically, the authors call for the creation of focused consortia to address specific scientific problems: identifying the immune correlates of protection in animal models, characterizing acute infection in humans, developing vaccines that can induce neutralizing antibodies and T-cell responses and systematically testing adjuvants.

CHAVI stems from these recommendations. It will initially focus on elucidating correlates of protection and/or early immunologic and virologic events after HIV infection, as well as initiating the evaluation of vaccine candidates. This month, a panel of $\mathrm{NIH}$-selected reviewers is considering applications from small teams of scientists—extensive research proposals outlining goals for the seven-year period, with a more detailed plan for the first year. The selected team will form the scientific leadership committee that will shape all CHAVI research activities and recruit other collaborators to work on defined projects. The awardees will be announced by the end of this September.
Organization and cooperation are clearly needed to design and evaluate vaccine trials. With multiple candidates entering the vaccine pipeline and limited resources for testing them, the need for consensus on how to assess and compare different vaccine immunogens is evident. Steps are already being taken toward coordinating trials and formulating standardized assays to assess them. But, at the level of basic research, will simply providing new infrastructure advance our understanding of how to make an effective HIV vaccine? There is an element of paradox in the Enterprise's plan: its authors note that the main obstacles to the development of a vaccine are scientific, not organizational, yet they advocate organization to solve the scientific problems. The prescriptive CHAVI approach - a small number of researchers directing a predetermined program of research-may be unlikely to provide the elusive conceptual breakthroughs that are sorely needed.

There are, however, reasons to support such a coordinated research structure. CHAVI may not provide the breakthrough, but by providing more resources than are available to individual groups, by outlining clear goals and endpoints, and by forging synergy between scientists with complementary expertise, it will probably speed the resolution of questions that are widely recognized as important. And there are precedents for believing that such an approach will lead to some good science. An NIHfunded collaborative venture started in 1993 that also aimed to determine the correlates of HIV immune protection may not have resulted in an effective vaccine, but the collaboration did result in many fundamental insights into viral dynamics, viral evolution and host factors involved in protection from infection in persistently exposed individuals.

But that particular venture received around $\$ 13$ million in total funding over four years-a fraction of the anticipated CHAVI budget. If it is possible to drive productive collaborations on much less than $\$ 300$ million, as seems evident, a more prudent approach this time around might have been to fund multiple projects, each with a smaller budget.

Ultimately, until the successful applicants-and the scientific details of their proposal - are announced, it is premature to predict whether the initiative will succeed in driving the field forward. But with such a huge sum at stake, the architects of CHAVI must plan for some external oversight and clear measures of value for money. It is equally crucial that the creativity of individual researchers is allowed to flourish. The funding earmarked for the first year of CHAVI activities will not affect the RO1 pool, but the source for the next six years is not clear. Given the present climate of tightening federal purse strings, the $\mathrm{NIH}$ should guarantee that future funding for CHAVI does not come at the expense of RO1 grants. 